\title{
Effects of early alcohol exposure upon adult learning ability and taste preferences
}

\author{
DAVID S. PHILLIPS and GENE L. STAINBROOK \\ Department of Medical Psychology \\ University of Oregon Health Sciences Center, Portland, Oregon 97201
}

The purpose of this study was to determine the effect of preweaning exposure to alcohol upon adult learning ability and taste preferences. Two groups of rats, one whose mothers drank only water and one whose mothers drank only wine, were tested as adults for their ability to form learning sets and for their preference between water and wine. Animals whose mothers drank wine performed below the level of control animals with regard to learning set formation and consistently drank more wine than the controls.

The purpose of this study was to investigate the effects of early exposure to alcohol upon the learning ability and taste preferences of adult organisms. Several investigators have reported cases involving human infants born to alcoholic mothers (Bianchine \& Taylor, 1974; Ferrier, Nicol, \& Ferrier, 1973; Hall \& Orenstein, 1974; Jones \& Smith, 1973; Jones, Smith, Streissguth, \& Myrianthopoulos, 1974). Typically, these infants have small palpebral fissures, microphthalamia, epicanthal folds, and cardiac anomolies and are small in terms of height, weight, and head circumference (Jones, Smith, Ullelund, \& Streissguth, 1973). They also display retarded postnatal development both physically and behaviorally. The phrase, "fetal alcohol syndrome," FAS, has been used to describe this condition. The number of reported cases of FAS is small relative to the total number of alcoholic women, and since most of the women involved were older, multigravida, and hence more prone to bear offspring with congenital defects, the causal role played by alcohol has been questioned.

In order to investigate the question of whether prenatal or early postnatal exposure to alcohol influences an organism's ability to learn in adulthood, the ability of rats to form learning sets was investigated. Rats were chosen since it has been shown that they are sensitive to development perturbations when administered substances prenatally (Butcher, Vorhees, \& Kimmel, 1972; Golter \& Michaelson, 1975; Havlena \& Werboff, 1963). The ability to form learning sets has been taken by many investigators to be a higher order type of learning and therefore might be expected to be more sensitive in showing a deficit in learning ability. Furthermore, others have shown this ability to be susceptible to early insult (Waisman \& Harlow, 1965).

A second question under study was whether early exposure to alcohol influences an organism's preference for this substance at some later point in time. Galif and Henderson (1972) have demonstrated that rat pups show a preference for the diet that their mother has been fed even though the pups themselves have never consumed that diet. Supposedly, something is passed to the pups through the mother's milk that helps them in this selection. Certainly, alcohol consumed by the mother may also enter her milk and be passed to her pups. Whether this would have any effect, positively or negatively, on the pups self-selection of alcohol in adulthood was open to question.

\section{METHOD}

\section{Apparatus}

The apparatus used in this study was a WGTA designed for visual discrimination testing with rats. It consisted of a $31-\mathrm{cm}-$ high $\times 31-\mathrm{cm}$-wide $\times 41.5$-cm-long box with four 5 -cm-square holes cut in one end. Clear Plexiglas stimulus doors hinged from above were mounted on the outside of the box so that they covered these openings. A foodwell was located immediately behind each door. Two guillotine doors were mounted inside the box, a transparent one $10 \mathrm{~cm}$ from the stimulus doors and an opaque one immediately in front of the doors. The box was painted flat black and illuminated by a $15-\mathrm{W}$ fluorescent bulb mounted $15 \mathrm{~cm}$ behind the stimulus doors.

The visual stimuli consisted of 10 sets of patterns reported previously (Phillips, 1968). They were cut from black construction paper, mounted on white paper, and sealed in plastic. For a given set of stimuli, three were identical and the fourth, the correct one, was different. Within each set, the stimuli were equated for area to eliminate brightness cues.

\section{Subjects}

Six female hooded rats were obtained from one litter and at 60 days of age randomly divided into two groups. Group 1 animals were given only tap water to drink and Group 2 animals were given only chablis wine to drink. All animals had continuous access to rat pellets and their respective drinking fluids. A stud male was randomly selected from the colony, and the six females were mated after reaching 120 days of age. The six females were maintained on their respective drinking fluids during mating, pregnancy, and lactation. The offspring of these six females served as subjects in this study.

\section{Procedure}

The pups were weaned and weighed at 25 days of age, and one litter from each group was randomly selected for further study. Both litters were reduced to nine animals each. All animals were maintained on ad-lib water and food after weaning and were weighed at 50,75 , and 160 days of age. 
At 90 days of age, the animals were placed on a restricted diet and introduced to the test apparatus. Each animal received 10 days of pretraining so that by 100 days of age he was required to make 25 responses, a response being to nose open one of the stimulus doors and retrieve a $45-\mathrm{mg}$ Noyes pellet. Pretraining was conducted with blank pieces of white cardboard attached to the stimulus doors.

On the 101st day, one set of stimuli was randomly selected to serve as a training problem, and training began. Training consisted of placing the animal in the apparatus with all the foodwells baited, the stimulus doors in place, and both guillotine doors lowered. The opaque door was raised to allow the animal to observe the stimuli for $10 \mathrm{sec}$, and then the transparent door was raised. If the animal made a correct response, both guillotine doors were lowered as soon as he had obtained his pellet; if an incorrect response was made, both doors were lowered immediately before another response could be attempted. When both doors had been lowered, the stimuli were positioned for the next trial and the sequence was repeated. The position of the correct door was determined by a random sequence, with the restrictions that the correct stimulus appear at each door 25 times in 100 trials. Each animal was given 25 trials per day until it made 13 or more correct responses on 2 out of 3 consecutive days.

The other nine sets of stimuli were randomly arranged in a sequence, and when an animal reached criterion on the training problem he started on the test sequences. Each animal was given 25 trials a day for 4 days (100 trials) on each test problem. When an animal had finished all nine test problems, he was again given ad-lib access to food.

When the animals were 170 days of age, each was given a 5-day taste preference test between wine and water. This was done by mounting two $90-\mathrm{cc}$ drinking tubes on each cage, one containing tap water and the other containing chablis. The amount of each fluid consumed was measured daily, and the locations of the fluids were altered.

\section{RESULTS}

The three animals on tap water had litters of 11,11 , and 12 pups; those on wine had litters of 9,12 , and 13 pups. The mean weights for each group at $25,50,75$, and 160 days of age are given in Table 1 . The two groups differed significantly in mean weight at 25 days of age $(t=11.54, p \leqslant .001)$, and although the animals whose mothers had been on wine still weighed less at 50 days, this difference was not significant.

One animal in the Wine group died 3 days after being put on restricted food intake. The mean percent correct responses for each group on each of the nine test problems is given in Table 2. Both groups show improvement in performance across problems, demonstrating the formation of learning sets; however, the
Table 1

Mean Weight for Wine and Control Groups at 25, 50, 75, and 160 Days of Age

\begin{tabular}{|c|c|c|c|c|}
\hline & \multicolumn{4}{|c|}{ Age in Days } \\
\hline & 25 & 50 & 75 & 160 \\
\hline$\underline{\bar{X}}$ wine & 45.1 & 167.8 & 240.9 & 267.9 \\
\hline$\overline{\mathrm{X}}$ water & 66.7 & 184.9 & 228.6 & 262.8 \\
\hline $\mathrm{t}$ test & 11.54 & 1.27 & .65 & .20 \\
\hline df & 66 & 16 & 16 & 15 \\
\hline
\end{tabular}

Wine group surpasses the Water group on only two problems out of the nine. A one-tailed Wilcoxon matched pairs test applied to these data indicates significance $(T=7, p \leqslant .05)$.

The results of the taste preference tests are given in Figure 1. The Wine group consistently had a larger total daily fluid intake (a). Part b of Figure 1 indicates that the two groups drank essentially the same volume of water each day, so the difference was in the amount of wine consumed. Part $c$ of Figure 1 shows the mean daily wine intake for both groups.

\section{DISCUSSION}

The litter sizes of the two groups were essentially the same and none of the pups had any noticeable birth defects. Both sets of dams engaged in normal maternal behavior, and there were no instances of cannibalization.

The pups born to mothers who were drinking only wine weighed significantly less at weaning than those born to females which were drinking only tap water. By 50 days of age, this difference in weight was no longer significant. Fluid consumption of the dams was measured for 5 days before mating and was not significantly different. The mean weights for the dams on the day they were mated were: $271.3 \mathrm{~g}$ for the controls and $262.7 \mathrm{~g}$ for the Wine group. Hence the weight differences in the pups appears to be attributable to the fluids consumed by the mothers rather than the amount of fluid.

Both groups of animals displayed the formation of learning sets, but on seven of the nine test problems, the Wine group performed below the level of the Water group. Those rats born to mothers who drank only wine weighed less at weaning and formed learning sets more

Table 2

Mean Percent Correct Responses for Wine and Control Groups on Nine Test Problems

\begin{tabular}{|c|c|c|c|c|c|c|c|c|c|}
\hline & \multicolumn{9}{|c|}{ Problem } \\
\hline & 1 & 2 & 3 & 4 & 5 & 6 & 7 & 8 & 9 \\
\hline$\overline{\mathrm{X}} \%$ correct water & 59.8 & 63.3 & 73.5 & 82.9 & 86.9 & 92.1 & 93.4 & 93.4 & 93.8 \\
\hline & 15.5 & 18.3 & 18.4 & 8.2 & 8.2 & 5.2 & 3.1 & 5.1 & 5.2 \\
\hline$\overline{\mathrm{X}} \%$ correct wine & 50.1 & 61.0 & 73.4 & 79.6 & 87.4 & 90.5 & 93.0 & 94.0 & 92.5 \\
\hline SD & 12.5 & 14.5 & 11.6 & 6.7 & 7.8 & 7.0 & 6.1 & 4.5 & 4.3 \\
\hline
\end{tabular}



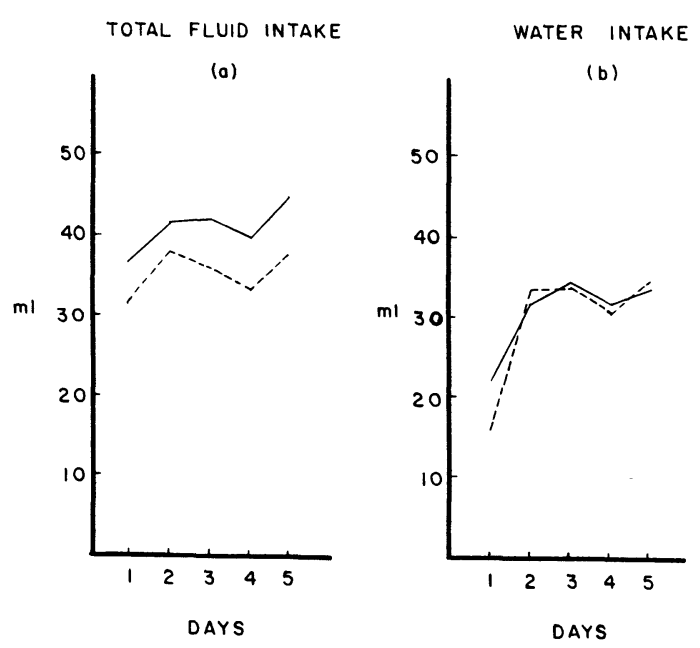

slowly than control animals. The fact that the weight differences did not persist longer, that no birth defects were noted, or that the effect on learning was not more severe might be attributable to the amount of alcohol in the mothers' systems. Before becoming pregnant, the three females drinking wine had a mean weight of $263 \mathrm{~g}$ and a mean daily wine intake of $44.8 \mathrm{ml}$, but blood alcohol levels were not determined for these animals, so it is possible that by spacing their drinking they may have been able to maintain fairly low blood alcohol levels over a 24-h period (Samson \& Falk, 1975).

During taste preference testing, the animals whose mothers drank wine consistently had a higher daily fluid intake than the control animals. When consumption was broken down into the amount of water and wine drunk, it was found that the two groups consumed the same amount of water and that the difference in total fluid consumption was due to the fact that the Wine group drank more wine. While the Wine group's consumption of wine never approached $50 \%$ of their daily fluid intake, it is certainly clear that a minimal early exposure to wine has increased their preference for this fluid over that of animals lacking this early exposure. Whether this preference is a function of the alcohol being passed to the pups through their mothers' milk or through direct consumption of small amounts of wine during the last few days before weaning cannot be determined from the present study. Whether a few days of drinking only wine after weaning would have increased this preference for wine in adulthood is another interesting but unanswered question.

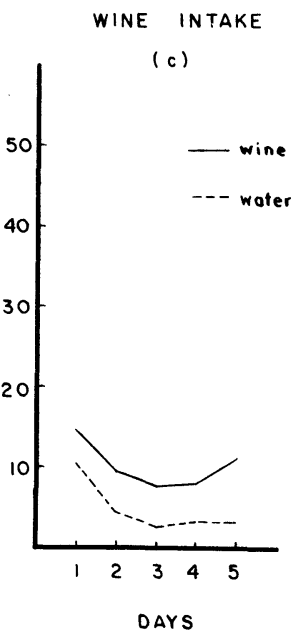

Figure 1. Mean daily fluid consumption.

\section{REFERENCES}

Bianchine, J. W., \& TAYlor, B. D. Noonan syndrome and fetal alcohol syndrome. Lancet, 1974, 1, 933.

Butcher, R. E., Vorhees, C. V., \& Kimmel, C. A. Learning impairment from maternal salicylate treatment in rats. Nature New Biology, 1972, 236, 211-212.

Ferrier, P. E., Nicol, I., \& Ferrier, S. Fetal alcohol syndrome. Lancet, 1973, 2, 1496.

GAlef, B. E., \& Henderson, P. W. Mother's milk: A determinant of the feeding preferences of weaning rat pups. Journal of Comparative and Physiological Psychology, 1972, 78, 213-219.

Golter, M., \& Michaelson, I. A. Growth, behavior, and brain catecholimines in lead-exposed neonatal rats: A reappraisal. Science, 1975, 187, 359-361.

HAll, B. D., \& ORenstein, W. A. Noonan's phenotype in an offspring of an alcoholic mother. Lancet, 1974, 1, 680-681.

Havlena, J., \& Werboff, J. Postnatal effects of control fluids administered to gravid rats. Psychological Review, 1963, 12 127-131.

Jones, K. L., \& SMith, D. W. Recognition of the fetal alcohol syndrome in early infancy. Lancet, 1973, 2, 999-1001.

Jones, K. L., Smith, D. W., Streissguth, A. P., \& Myrianthopoulos, N. C. Outcome in offspring of chronic alcoholic women. Lancet, 1974, 11, 1076-1078.

Jones, K. L., Smith, D. W., Ullelund, C. N., \& Streissguth, A. P. Pattern of malformation in offspring of chronic alcoholic mothers. Lancet, 1973, 1, 1267-1271.

Phillips, D. S. Olfactory cues in visual discrimination problems. Physiology and Behavior, 1968, 3, 683-685.

SAmson, H. H., \& FALK, J. L. Patterns of daily blood ethanol elevation and the development of physical dependence. Pharmacology Biochemistry and Behavior, 1975, 3, 1119-1123.

WAISMAN, H. H., \& HARLOW, H. F. Experimental phenylketonuria in infant monkeys. Science, 1965, 147, 685-695.

(Received for publication June 28, 1976; revision accepted August 13, 1976.) 